\title{
QUANTO MAIS SE SABE, MAIS SERGURANÇA TEM! A IMPORTÂNCIA DA GESTÃO DO CONHECIMENTO NA CONDUÇÃO DOS RESÍDUOS SÓLIDOS DE QUIMIOTERAPIA
}

DOI: 10.22289/2446-922X.V6N2A28

\author{
Layze Amanda Leal Almeida ${ }^{1}$ \\ Nilton S. Formiga \\ José Itamar A. Junior \\ Patrício Marques de Sousa
}

\section{RESUMO}

A quimioterapia é descrita com o uso de composições químicas sintéticas no combate aos agentes, sendo que, no combate ao câncer, sua atuação intervém diretamente na cinemática do tumor. Os resíduos provindos da quimioterapia são classificados como Resíduos dos Serviços de Saúde, sendo assim, se faz necessário controle e planejamento do gerenciamento destes tendo em vista sua origem, e sua periculosidade, por apresentar características de toxicidade, patogenicidade, carcinogenicidade, teratogenicidade e mutagenicidade, Resíduos Sólidos são aqueles que se encontram nos estados sólido e semi-sólido resultantes de atividades de diversas origens, incluindose também determinados líquidos cujas particularidades inviabilizam o lançamento na rede pública de esgotos ou corpos de água. O objetivo desta revisão bibliográfica é refletir a respeito da influência da gestão do conhecimento e oportunidade aprendizagem organizacional em relação ao manuseio dos resíduos dos serviços de saíde de quimioterapia pelos funcionários envolvidos no seu setor de trabalho. A pesquisa foi realizada a partir da busca de artigos nas bases de dados digitais Medline, Lilacs, Scielo e periódicos CAPES, utilizando palavras-chaves sobre o tema. A gestão de conhecimento ainda não possui uma estrutura universal ou uma metodologia bem estabelecida. $O$ processo de gestão de conhecimento é caracterizado como uma forma de promover a capacidade do indivíduo ou da coletividade de organizar, criar, incorporar, partilhar e integrar os conceitos de forma consistentes. A Gestão do Conhecimento associada à Oportunidade de Aprendizagem pode ser uma grande ferramenta para a gestão dos Resíduos de Serviços de Saúde de quimioterapia, assim como para um maior aprendizado, aceitação e busca pelo conhecimento por parte dos funcionários que lidam diretamente com os resíduos supracitados.

Palavras-chave: Gestão do Conhecimento; Resíduos dos Serviços de Saúde; Quimioterapia; Meio Ambiente.

\section{THE MORE YOU KNOW, THE MORE SAFETY YOU HAVE! THE IMPORTANCE OF KNOWLEDGE MANAGEMENT IN CONDUCTING CHEMOTHERAPY SOLID WASTE}

\section{ABSTRACT}

\footnotetext{
${ }^{1}$ Endereço eletrônico de contato: layzeala@gmail.com

Recebido em 10/11/2020. Aprovado pelo conselho editorial para publicação em 03/12/2020.
}

Rev. Psicol Saúde e Debate. Dez., 2020:6(2): 430-442. 
Solid wastes are those that are found in solid and semi-solid states, resulting from activities of industrial, domestic, hospital, commercial, agricultural, service and sweeping origin. Also included are certain liquids whose particularities prevent the introduction into the public network of sewage or water bodies, as it causes risks to the environment, society and public health, thus requiring technically and economically unviable solutions in view of the best available technology. The purpose of this literature review is to reflect on the influence of knowledge management and organizational learning opportunity in relation to the management of RSS of chemotherapy by employees involved in their work sector. The research was carried out from the search of articles in the digital databases Medline, Lilacs, Scielo and CAPES journals, using keywords within the subject. Knowledge management does not yet have a universal framework or a well-established methodology. Several authors that the process of knowledge management is characterized as a way to promote the ability of the individual or the community to organize, create, incorporate, share and integrate concepts in a consistent way. Knowledge Management associated with the Learning Opportunity can be a great tool for the management of the RSS of chemotherapy, as well as for a greater learning, acceptance and search for knowledge by the employees who deal directly with the abovementioned residues.

Keywords: Knowledge Management; Waste from Health Services; Chemotherapy; Environment.

\section{¡CUANTO MÁS SEPA, MÁS SEGURIDAD TENDRÁ! LA IMPORTANCIA DE LA GESTIÓN DEL CONOCIMIENTO EN LA REALIZACIÓN DE RESIDUOS SÓLIDOS DE QUIMIOTERAPIA}

\section{RESUMEN}

La quimioterapia se describe con el uso de composiciones químicas sintéticas en la lucha contra los agentes, y en la lucha contra el cáncer, su acción interviene directamente en la cinemática tumoral. Los residuos de la quimioterapia se clasifican como Residuos de los Servicios de Salud, por lo tanto, es necesario controlar y planificar su manejo en vista de su origen y su peligrosidad, ya que presentan características de toxicidad, patogenicidad, carcinogenicidad, teratogenicidad y mutagenicidad. Los residuos sólidos son aquellos que se encuentran en estado sólido y semisólido como resultado de actividades de diferentes orígenes, incluidos ciertos líquidos cuyas particularidades hacen imposible la descarga al alcantarillado público o cuerpos de agua. El propósito de esta revisión bibliográfica es reflexionar sobre la influencia de la gestión del conocimiento y la oportunidad de aprendizaje organizacional en relación con el manejo de los desechos de los servicios de quimioterapia de salud por parte de los empleados involucrados en su sector de trabajo. La investigación se llevó a cabo buscando artículos en las bases de datos digitales de las revistas Medline, Lilacs, Scielo y CAPES, utilizando palabras clave sobre el tema. La gestión del conocimiento aún no tiene una estructura universal o una metodología bien establecida. El proceso de gestión del conocimiento se caracteriza por promover la capacidad individual o colectiva de organizar, crear, incorporar, compartir e integrar conceptos de manera coherente. La Gestión del Conocimiento asociada con la Oportunidad de Aprendizaje puede ser una gran herramienta para la gestión de los Residuos de Servicios de Salud de la quimioterapia, así como para un mayor aprendizaje, aceptación y búsqueda de conocimiento por parte de los empleados que se ocupan directamente de los residuos antes mencionados.

Palabras clave: Gestión del conocimiento; Desperdicio de Servicios de Salud; Quimioterapia; Medio ambiente.

Rev. Psicol Saúde e Debate. Dez., 2020:6(2): 430-442. 


\section{INTRODUÇÃO}

Resíduos sólidos são aqueles que se encontram nos estados sólido e semissólido, resultantes de atividades de origem industrial, doméstica, hospitalar, comercial, agrícola, de serviços e de varrição. Incluem-se também determinados líquidos cujas particularidades inviabilizam o lançamento na rede pública de esgotos ou corpos de água, por causar riscos ao meio ambiente, sociedade e a saúde pública, exigindo, portanto, soluções técnicas e economicamente inviáveis tendo em vista a melhor tecnologia disponível (NBR, 2004).

Os resíduos dos serviços de saúde (RSS) são aqueles produzidos em hospitais, laboratórios, consultórios odontológicos, clínicas médicas e veterinárias, parte importante do total de resíduos sólidos urbanos, não pela quantidade gerada (cerca de 1\% a $3 \%$ do total), mas pelo risco que representam à saúde e ao meio ambiente. Dentre estes destaca-se os resíduos sólidos de quimioterapia, pois apresentam risco potencial à saúde pública e ao meio ambiente devido às suas características químicas e biológicas (Brasil, 2006).

É certamente um grande paradoxo do desenvolvimento científico e tecnológico os conflitos com os quais se depara o homem contemporâneo diante dos graves e inúmeros problemas ambientais e sanitários provenientes da ação de sua própria criatividade e inovação. Em meio a estes, encontram-se aqueles gerados pelo descarte inadequado de resíduos que, por conseguinte, criam enormes passivos ambientais, pondo em risco toda a qualidade de vida das gerações presentes e futuras, além de causar graves problemas ambientais. $O$ acondicionamento inadequado decorrente de ações de agentes químicos, físicos e biológicos, cria condições ambientais potencialmente perigosas, onde modificam-se os agentes e propiciam sua disseminação ambiental, afetando diretamente a saúde humana (Brasil, 2006).

O gerenciamento inadequado dos resíduos de saúde pode atingir grandes proporções que vão desde as pequenas contaminações e elevados níveis de infecção hospitalar até o desencadeamento de epidemias/endemias devido à contaminação do lençol freático por diversos tipos de RSS.

Muitos segmentos empresariais já avançaram neste contexto, porém a área de saúde necessita de iniciativas, estudos e projetos eficientes que contribuam para a mudança da realidade, de modo que a preocupação com o desenvolvimento sustentável seja um dos caminhos para manutenção e melhoria de vida das pessoas.

Com a transição epidemiológica no Brasil e no mundo houve crescimento das doenças crônico-degenerativas, incluindo as afecções oncológicas, que tem produzido, consequentemente, aumento dos resíduos provenientes da administração dos medicamentos quimioterápicos.

No início do século 20 Paul Erlich criou o termo quimioterapia, objetivando descrever o uso de composições químicas sintéticas no combate aos agentes infecciosos (3). No combate ao Rev. Psicol Saúde e Debate. Dez., 2020:6(2): 430-442. 
câncer, o termo quimioterapia ganha um adjunto adnominal antineoplásica ou antiblática, tendo em vista que sua atuação irá ocorrer nos processos de crescimento e divisão celular ativos (mitoses), desse modo, intervindo diretamente na cinemática do tumor (Brasil, 2009).

Os resíduos provindos da quimioterapia, isto é, os quimioterápicos (QA), são classificados como RSS, tendo em vista sua origem, e sua periculosidade, por apresentar características de toxicidade, patogenicidade, carcinogenicidade, teratogenicidade e mutagenicidade, devendo, com isso, estar regulamentado junto ao Sistema Nacional do Meio Ambiente (SISNAMA) e Sistema Nacional de Vigilância Sanitária (SNVS) (Brasil, 2010).

Sabendo-se que a quimioterapia se embasa na administração de vários tipos de medicações antineoplásicas, se faz necessário controle e planejamento do gerenciamento destes RSS que são eliminados pelo setor da assistência quimioterápica, tendo em vista que estas medicações são constituídas de substancias químicas/biológicas que se não manejadas e acondicionadas corretamente poderão trazer sérios danos aos profissionais que lidam diretamente com as mesmas, além de trazer riscos gravíssimos ao meio ambiente e a qualidade de vida de toda uma população.

Com isso, acredita-se que o presente artigo tem sua relevância, por associar os referidos construtos referentes a Gestão de Conhecimento e aprendizagem organizacional com o RSS (mais especificamente de quimioterapia), com ênfase na necessidade de uma gestão eficiente e o cuidado com meio ambiente e qualidade de vida dos profissionais envolvidos neste cenário.

Frente a essas condições questiona-se: seria este problema exclusividade de uma gestão normativa sobre os Resíduos dos Serviços de Saúde (RSS) ou é possível inserir neste espaço ambiental o sujeito e a instituição responsáveis pelo manuseio e descarte desses materiais? Sendo assim, o objetivo central desse artigo trata-se de refletir a respeito da influência da gestão do conhecimento e oportunidade aprendizagem organizacional em relação ao manuseio dos RSS de quimioterapia pelos funcionários envolvidos no seu setor de trabalho.

\section{DESENVOLVIMENTO}

Observou-se não apenas uma carência de estudos do tipo quali-quantitativos ou especificamente, qualitativos e quantitativos, referentes a Resíduos Sólidos de Saúde (RSSS) das unidades de quimioterapia sob a óptica da saúde e do meio ambiente, bem como, do RSS e sua relação com a gestão do conhecimento e aprendizagem organizacional, especialmente, no território paraibano, e mais específico, no estado da Paraíba-PB.

A geração e a manipulação inadequada dos RSS no meio ambiente podem colaborar para poluições físicas, biológicas e químicas do solo, da água e do ar, submetendo o ser humano as

Rev. Psicol Saúde e Debate. Dez., 2020:6(2): 430-442. 
mais variadas formas de exposição ambiental. O gerenciamento inadequado dos mesmos nos estabelecimentos de saúde vem gerando aumento significativo de profissionais acometidos por acidentes de trabalho, além de ser importante fator contribuinte para a ampliação na incidência de infecções hospitalares (Hospital Universitário Júlio Muller - HUJM, 2004).

Desse modo, para propiciar a diminuição dos riscos à saúde pública, proteção dos trabalhadores e a preservação do meio ambiente, se faz mister o bom gerenciamento dos resíduos sólidos nos estabelecimentos de saúde por meio da implantação de um Plano de Gerenciamento de Resíduos nos Serviços de Saúde (PGRSS) nas instituições de saúde, este tem como finalidade o manejo seguro dos resíduos, e por conseguinte, o cumprimento das normas referentes às características químicas, físicas e biológicas dos materiais, vislumbrando todas as etapas do processo, desde a segregação dos resíduos até o seu destino final (Silva Junior, 2007).

Entende-se como manejo integrado dos Resíduos de Serviços de Saúde a ação de gerenciálos em seus aspectos intra e extra estabelecimento, desde o momento de geração até a sua disposição final, incluindo todo o processo: segregação, acondicionamento, identificação, coleta, transporte interno, tratamento preliminar, armazenamento temporário e externo e tratamento final ((Hospital Universitário Júlio Muller - HUJM, 2004).

A Agência Nacional de Vigilância Sanitária - ANVISA e o Conselho Nacional do Meio Ambiente - CONAMA vêm assumido no Brasil um papel importante de orientar, definir regras e regular a conduta dos diferentes agentes, no que se refere à geração e ao manejo dos RSS, objetivando a preservação da saúde e do meio ambiente, e garantindo a sua sustentabilidade.

Desde o início da década de 90, procedem empregando esforços no sentido do correto gerenciamento dos resíduos de serviços de saúde e da responsabilização do gerador. Aa publicação da Resolução CONAMA nº 005/93, que determinou a obrigatoriedade aos serviços de saúde da elaboração do Plano de Gerenciamento de seus resíduos mostrou-se como o primeiro passo significativo na legislação relativa ao gerenciamento de RSS, que repercutiu em outras publicações (RDC ANVISA n 306/04; CONAMA $n^{\circ}$ 358/05) as quais reafirmaram o compromisso destas instituições com a problemática do manejo dos resíduos nas instituições de saúde (Brasil, 2006).

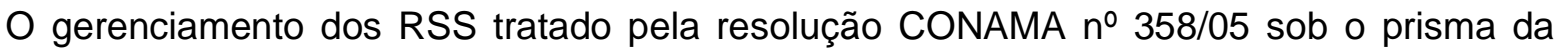
preservação dos recursos naturais e do meio ambiente, promove a competência aos órgãos ambientais estaduais e municipais para estabelecerem critérios de licenciamento ambiental dos sistemas de tratamento e destinação final dos RSS (Brasil, 2006).

Em complemento a RDC ANVISA nº 306/04 emprega sua regulação no controle do manejo: segregação, acondicionamento, armazenamento, transporte, tratamento e disposição final. Constituindo procedimentos operacionais em função dos riscos envolvidos, de modo a concentrar seu controle na inspeção dos serviços de saúde (Brasil, 2006).

Rev. Psicol Saúde e Debate. Dez., 2020:6(2): 430-442. 
A RDC ANVISA no 306/04 e a Resolução CONAMA no 358/2005, definem como geradores de RSS todos os serviços relacionados com o atendimento à saúde humana ou animal (Brasil, 2006). Portanto, o RSS decorrentes do tratamento sistêmico do câncer que usa medicamentos denominados genericamente de "quimioterápicos" (sejam eles quimioterápicos propriamente ditos, hormonioterápicos, bioterápicos, imunoterápicos, alvoterápicos) administrados continuamente ou a intervalos regulares, variando de acordo com os esquemas terapêuticos, caracterizam-se como resíduos os quais a manipulação e descarte encontra-se sob regulamentação das resoluções supracitadas (Brasil, 2010).

Considerando que essas normas e diretrizes não funcionam no 'vazio social', mas que se trata de uma orientação para que instituição e funcionários que lidam do RSS precisem melhor gerir comportamentos de segurança pessoal e coletiva, acredita-se na importância de que instituição e funcionários possam trabalhar na direção para que ambos sejam esclarecidos e consciente dos valor de um comportamento socialmente desejável para a proteção do seu entorno social e ambiental.

\subsection{Gestão do conhecimento em saúde e sua função no contexto hospitalar}

Há duas décadas surgiu a Gestão do Conhecimento, e a partir disso, neste mesmo período, vem se mostrando como um tema de interesse expressivo em diversas áreas cientificas (Administração, Psicologia e Sociologia da organização, Enfermagem, etc.), tanto no meio acadêmico quanto no meio organizacional. Fato este que vem acontecendo como resultado da estreita relação que a maioria dos autores estabelece entre os processos organizacionais relacionando-os com o conhecimento e por conseguinte com o desempenho organizacional, inovação, criatividade (Cardoso, 2003; Fandiño Formiga, \& Menezes, 2018).

Apesar da valorização do tema da gestão em distintas áreas da ciência humana, social e da saúde, esta, não poderá ser concebida especificamente, uma variável isolada, do tipo apenas descritivo em termos da existência de uma frequência e intensidade sobre a melhor forma de gerir conhecimento no ambiente de trabalho, mas, deve-se considerar a importância dessa gestão no capacidade e desenvolvimento de um aprendizagem destinada ao espaço laboral; é neste sentido que se considera que a aprendizagem organizacional, além de complexa e dinâmica quando comparada a aprendizagem individual, tem a função de ampliar tal processo ensino-aprendizagem na empresa e no capital humano, pois é capaz de alcançar uma perspectiva grupal no binômio organização-funcionário (Kim, 1993).

Desta forma, com uma abrangência maior que o capital intelectual e mais operacional, e do conceito de organização aprendiz, a gestão de conhecimento, mesmo mostrando variações quanto as atribuições conceituais por parte de diferentes autores, esta, corresponde portanto, a todo um Rev. Psicol Saúde e Debate. Dez., 2020:6(2): 430-442. 
esforço sistemático que a organização emprega na sua estrutura interna e relacional com os funcionários a fim de desenvolver, utilizar, reter e mensurar o conhecimento de ambos (organização e trabalhador) (Loureiro, 2003).

No que se refere a aplicabilidade do tema sobre a gestão de conhecimento e que ela seja objeto de interesse por vários setores de trabalho, só recentemente passou a receber o interesse do setor da saúde (Kothari, Hovanec, Hastie, \& Sibbald, 2011).

Neste setor, a implementação deste construto (por exemplo, gestão do conhecimento) configura-se pertinente, aspecto que advém, por um lado, do fato das instituições de saúde serem de conhecimentos intensivos e, por outro, na sequência das sucessivas medidas reformistas que os Sistemas de Saúde (Europeus, Português e Brasileiro), nos quais, buscando um controle dos custos e na procura por uma maior eficiência, num setor, o qual caracteriza-se cada vez mais num cenário de competitividade e de inovação. Apesar das medidas implementadas não produzirem, muitas vezes, os resultados desejados para a população e, também, para os seus funcionários, exigindo com isso, um empenho contínuo em busca de uma reforma e otimização do cenário dos serviços de saúde (Mourão, 2009; Santos Júnior, Bispo, \& Abbad, 2014).

Desse modo, num cenário onde se generalizam novas estratégias de reforma dos setores públicos de saúde, embasados nos princípios de Nova Gestão Pública, e que assemelhar-se na competição para promover a eficiência, a gestão do conhecimento assume um papel significativo, com vistas na concretização da ambição em questão, bem como, gerir fatores de proteção funcional e de saúde individual e coletiva (Ferreira, 2005).

Reformas e inovações na gestão das instituições de saúde têm tido uma questão central na política de saúde contemporânea, correspondendo assim, à necessidade de tomada de consciência de que muitos dos problemas, com os quais organizações vivenciam cotidianamente, não encontram respostas nos modelos tradicionais de gestão, sendo as soluções insuficientes/ ineficazes, as quais, podem acarretar problemas mais graves na população (por exemplo, contaminação do solo, das águas, etc.) (Cruz \& Ferreira, 2017).

Diante deste tema, é necessário que a estrutura organizacional e a cultura organizacional que compõem variáveis reconhecidas como fatores limitantes do sucesso das mudanças organizacionais, nas instituições de saúde, aspecto que decorre sobretudo do fato destas instituições serem estruturalmente hierarquizadas e burocráticas, alicerçadas em valores tradicionais que atualmente se mostram não adequados, mas que continuam a influenciar muitas práticas e decisões, mesmo quando o discurso preconiza a adesão de outros de gestão (Neves, 2020).

Para isso, é que uma variável que contribuiria muito para avaliar a qualidade da gestão de conhecimento dos funcionários, seria da oportunidade de aprendizagem organizacional; esta, teria uma relação direta com as políticas de recursos humanos destinadas ao processo e resultado Rev. Psicol Saúde e Debate. Dez., 2020:6(2): 430-442. 
quanto a disponibilidade do aprender nas organizações capaz de influenciar na intenção de rotatividade, comprometimento e maior investimento emocional frente as demandas do trabalho (compreendido como capital psicológico positivo), condições essas que contribui tanto para a identificação das percepções sobre as oportunidades de trabalho quanto à aplicação de estratégias que fomentem melhorias na cultura, atitudes e comportamentos organizacionais (por exemplo, motivação, clima organizacional, satisfação com o trabalho, etc.) (Freitas, Lacerda, \& Santos, 2015; Cardoso, 2016).

Ainda de acordo com os autores supracitados, as oportunidades de aprendizagem, mesmo que estejam estruturadas sob aspectos formais e informais do aprender, elas contemplam um conjunto de ações e atores, capaz assimilar, compreender e aplicar desde uma informação dirigida para o processo de trabalho à informação que se destine ao desenvolvimento profissional.

\subsection{Oportunidade de Aprendizagem Organizacional}

Nos estudos da área organizacional tem sido comum uma diferenciação do aspecto cada vez mais completo relativo ao ambiente, tendo como pressuposto que a competição entre as organizações não se restrinja as questões geográficas. A necessidade de mudanças cada vez mais constantes também têm sido destacadas, tendo em vista a imprevisibilidade, impondo às organizações a necessidade do desenvolvimento de estratégias para que as mesmas se desenvolvam e mantenham-se competitivas (Bezerra, 2018).

Para o enfrentamento dos desafios que se deparam as organizações, as mesmas têm desenvolvido diferentes estratégias de enfrentamento. Entretanto, existe um fator de relevância e reconhecimento para o sucesso de uma organização, que é a sua força de trabalho (Bezerra, 2018).

Neste contexto, os colaboradores das organizações são peça fundamental para um bom desempenho organizacional e um respeitável fator de criação de riqueza para as empresas. Justifica-se assim a preocupação que vem surgindo quanto a forma de gerenciar o capital humano, de modo que haja uma promoção de comprometimento e envolvimento dos componentes da empresa (funcionários) (Edivinsson \& Malone, 1998; Vaz, Inomata, Viegas, Selig, \& Varvakis, 2015).

Diante do que já foi exposto, pode-se dizer que a aprendizagem organizacional tem como principal característica a forma como as organizações aprendem, se relaciona com o meio e como dele retira informações as quais incorpora no seu cotidiano, tendo como o objetivo manter-se em um desenvolvimento constante, de modo a agregar vantagens que as tornem competitivas (Scorsolinl-Comin, Inocente, \& Miura, 2011).

A aprendizagem organizacional é portanto, um processo pelo qual se avalia sempre as experiências e busca transformar o resultado dessas avaliações em conhecimento disponível para a organização. Assim sendo, as organizações só poderão por meio de indivíduos que aprenderam.

Rev. Psicol Saúde e Debate. Dez., 2020:6(2): 430-442. 
A partir disso se faz necessário o estímulo de novas formas de pensar, incentivando que as pessoas estejam em aprendizado continuo, tanto individual como coletivamente (Senge, 1991).

A teoria organizacional pós-contemporânea vem sendo caracterizada pelo paradigma, o qual conceitualiza a organização como um sistema de processamento de informações e resolução de problemas. Desse modo, torna-se fundamental para o desempenho de uma organização o quanto ela é eficiente em lidar com as informações colhidas e com as decisões que serão tomadas através delas, em um ambiente muitas vezes arraigado de incertezas (cf. Mendonça, 2018).

Vale salientar que a aprendizagem não pode ser reduzida a esforço isolado, como aquele promovido pelo próprio trabalhador $(29,30)$. No tocante a esses aspectos, uma organização precisa tornar viável as oportunidades de aprendizagem para seus funcionários, de modo que a partir da aprendizagem individual, a mesma torne-se coletiva e traga benefícios/transformações concretas para a organização na qual estiverem inseridos (Souza, 2004; Rasoulinezhad, 2011).

Desse modo, não adianta apenas focar as organizações no além das conceito de aprendizagem organizacional, tendo em vista que para que os benefícios possam acontecer, elas precisam promover a gestão do conhecimento. Seguindo a mesma linha de pensamento, a aprendizagem organizacional é uma ferramenta que vem a complementar a gestão do conhecimento (King, 2009; Jain \& Moreno, 2015).

Deve-se estimular um clima de confiança, parceria e troca de conhecimentos de forma mútua entre os funcionários e as suas respectivas organizações, pois assim poderá chegar aos ideais almejados pela gestão do conhecimento (33). Esse clima de confiança e "leveza" tem intrincado em si diversos aspectos, tanto os organizacionais quanto aos aspectos relacionados aos trabalhadores. A partir disso a confiança, encorajamento e comprometimento seriam variáveis possíveis de serem atendidas no tocante aos funcionários. Ainda no que diz respeito a organização, vale salientar que além de todos as características citadas anteriormente, o fator cultural necessita de destaque neste processo (Alvares, Baptista, \& Araújo Júnior, 2010; Cardoso, 2016).

Com isso, considerando o conceito de cultura organizacional, este, é enfático no que diz respeitos as normas, de modo a considerar especificamente a aprendizagem organizacional. Um ambiente considerado propício à aprendizagem deve ter como principais características a existência de normas que ofereçam uma certa previsibilidade a respeito do que acontece/acontecerá em uma organização e sobre qual o comportamento almejado pelos trabalhadores, de modo individual ou coletivo (Borgstede \& Lundqvist, 2006; Santos \& Souza, 2014).

A partir desta pesquisa, foi possível observar não apenas uma carência de estudos do tipo quali-quantitativos ou especificamente, qualitativos e quantitativos, referentes a Resíduos Sólidos de Saúde (RSSS) das unidades de quimioterapia sob a óptica da saúde e do meio ambiente, bem como, do RSS e sua relação com a gestão do conhecimento e aprendizagem organizacional, especialmente, no território paraibano, e mais específico, no estado da Paraíba-PB.

Rev. Psicol Saúde e Debate. Dez., 2020:6(2): 430-442. 


\section{CONSIDERAÇÕES FINAIS}

Por ser uma área considerada nova no âmbito do saber, a gestão de conhecimento ainda não possui uma estrutura universal ou uma metodologia bem estabelecida, assim sendo o que se percebe é a abordagem individual por alguns autores sobre esta temática.

É comum ver através dos diversos autores que o processo de gestão de conhecimento é caracterizado como uma forma de promover a capacidade do indivíduo ou da coletividade de organizar, criar, incorporar, partilhar e integrar os conceitos de forma consistentes. E nesse contexto a gestão se torna importante para o gerenciamento dos RSS de quimioterapia.

A Gestão do Conhecimento associada à Oportunidade de Aprendizagem pode ser uma grande ferramenta para a gestão dos RSS de quimioterapia, assim como para um maior aprendizado, aceitação e busca pelo conhecimento por parte dos funcionários que lidam diretamente com os resíduos supracitados.

É importante salientar que a capacitação continuada com vista na Oportunidade de Aprendizagem dos indivíduos que trabalham na quimioterapia, poderia trazer uma maior adesão dos funcionários à medidas preventivas (Equipamentos de Proteção Individual e Protocolos), ações que facilitem o seu dia a dia e a captação de conhecimentos individual e coletivo, gerando assim uma disseminação do conhecimento, uma maior valorização do profissional da instituição e como resposta um melhor cuidado prestado, onde pacientes e funcionários estariam satisfeitos e seguro diante de um setor de prestação de serviço de saúde tão complexo.

\section{REFERÊNCIAS}

Alvares L, Baptista S. G, \& Araújo Júnior R. H. (2010). Gestão do conhecimento: categorização conceitual. Em Questão 16 (2), 235-252. Disponível em: https://seer.ufrgs.br/EmQuestao/article/view/15124.

ABNT. (2004). Associação Brasileira de Normas Técnicas. NBR 10.0004: Resíduos Sólidosclassificação. Rio de Janeiro, RJ.

Bezerra J. W. P. (2018). Anomia, Gestão do Conhecimento e Percepção de Oportunidades de Aprendizagem Organizacional: Estudo com Trabalhadores Brasileiros. [Dissertação]. Rio de Janeiro: Universidade Federal do Rio de Janeiro

Borgstede, C. V., \& Lundqvist, L. J. (2006). Organizational Culture, Professional Role Conceptions and Local Swedish Decision-makers' Views on Climate Policy Instruments. Journal of Environmental Policy \& Planning, 8 (4), 279-292. Disponível em: https://www.researchgate.net/publication/241143638_Organizational_Culture_Professional_R

Rev. Psicol Saúde e Debate. Dez., 2020:6(2): 430-442. 
ole_Conceptions_and_Local_Swedish_Decisionmakers'_Views_on_Climate_Policy_Instruments.

Brasil. (2010). Departamento de Regulação, Avaliação e Controle. Manual de Bases Técnicas da Oncologia - Sia/Sus - Sistema De Informações Ambulatoriais. 11르 ed. Brasília: Ministério da Saúde.

Brasil. (2006). Ministério da Saúde. Agência Nacional de Vigilância Sanitária. Manual de Gerenciamento de Resíduos Sólidos de Serviços de Saúde. Brasília: Ministério da Saúde.

Brasil. (2009). Ministério da Saúde. Instituto Nacional do Câncer. Administração de Quimioterápicos. Brasília: Ministério da Saúde.

Cardoso, A. P. B. (2016). Capital psicológico e oportunidade de aprendizagem organizacional: um estudo de caso em uma instituição de ensino superior. [Monografia]. São Leopoldo: Universidade do Vale do Rio dos Sinos.

Cardoso, L. M. G. P. P. (2003). A Gerir conhecimento e gerar competitividade: estudo empírico sobre a gestão do conhecimento e o seu impacto no desempenho organizacional. [Tese]. Coimbra, Portugal: Universidade de Coimbra.

Cruz, S. G., \& Ferreira, M. M. F. (2017). Gestão do Conhecimento em Hospitais Públicos com Diferentes Modelos de Gestão: alguns fatores explicativos. Brazilian Journal of Management \& Innovation, 4 (2),1-20. Disponível em: https://www.researchgate.net/publication/319086760_Gestao_do_conhecimento_em_hospitai s_publicos_com_diferentes_modelos_de_gestao_alguns_fatores_explicativos.

Davenport, T., \& Prusak, L. (1998). Conhecimento Empresarial: como as organizações gerenciam seu capital intelectual. Rio de Janeiro: Campus.

Edivinsson, L., \& Malone, M. S. (1998). Capital intelectual. São Paulo: Makron Books.

Fandiño, A. M., Formiga, N. S., \& Menezes, R. M. (2018). Organizational social capital, resilience and innovation validation of a theoretical model for specialized workers. Journal of Strategy and ManagementT, 12 (1), 137-152 Disponível em: https://doi.org/10.1108/JSMA-05-2018-0041.

Ferreira, M. M. F. (2005). Empenhamento organizacional de profissionais de saúde em hospitais com diferentes modelos de gestão. [Tese]. Braga, Portugal: Universidade do Minho.

Freitas, A. D. G, Lacerda, F. M., Santos, E. A., \& Alvarenga, M. A. (215). Intenção de rotatividade: um enfoque a partir das políticas de recursos humanos e da percepção de oportunidades de aprendizagem nas organizações. Anais do XXXIX Encontro da ANPAD; $13-16$ set 2015; Belo Horizonte (MG).

Hospital Universitário Júlio Muller. (2004). Programa de Gerenciamento de Resíduos Serviços de Saúde. Setor de Serviços Gerais e CME. № 9. 18p. Disponível em: http://www.ufmt.br/ufmt/site/userfiles/file/hujm/Comissoes\%20e\%20Comites/Programa\%20de \%20gerenciamento\%20de\%20res\%C3\%ADduos\%20servi\%C3\%A7os\%20de\%20sa\%C3\%BA de.pdf.

Jain, A. K., \& Moreno, A. (2015). Organizational learning, knowledge management practices and firm's performance. The Learning Organization, 22 (1), 14-39. Disponível em: https://www.emerald.com/insight/content/doi/10.1108/TLO-05-2013-0024/full/html.

Rev. Psicol Saúde e Debate. Dez., 2020:6(2): 430-442. 
Kim, H. D. (1993). The Link Between Individual and Organizational Learning. Sloan Management Review, $\quad 35$ (1), 37-50. Disponível em: https://www.researchgate.net/publication/40960146_The_Link_Between_Individual_And_Orga nizational_Learning.

King, W. R. (2009). Knowledge Management and Organizational Learning Knowledge Management and Organizational Learning. Annals of Information Systems, 4 (1), 3-13. Disponível em: http://www.uky.edu/ gmswan3/575/KM_and_OL.pdf.

Kluge, J., Stein, W., \& Licht, T. (2002). Gestão do conhecimento. Cascais: Principia.

Kothari, A., Hovanec, N., Hastie, R., \& Sibbald, S. (2011). Lessons from the business sector for successful knowledge management in health care: a systematic review. BMC Health Services Research, 11 (1). Disponível em: http://www.biomedcentral.com/1472-696/11/173.

Loureiro J. L. (2003). Gestão do Conhecimento. Lisboa: Centro Atlântico.

Mendonça, L. (2018). Gestão do Conhecimento e Inovação. Editora Poisson: Belo Horizonte - MG: Poisson.

Mourão L. (2009). Oportunidades de qualificação profissional no Brasil: reflexões a partir de um panorama quantitativo. RAC, 13 (1),136-153. Disponível em: http://www.scielo.br/pdf/rac/v13n1/a09v13n1.pdf.

Neves A. (2002). Gestão na Administração Pública. Cascais: Pergaminho.

Nonaka I. (1992). A dynamic theory of organizational knowledge creation. Organization Science, 5 (1), 14-37. Disponível em: http://www.svilendobrev.com/1/Nonaka_1994Dynamic_theory_of_organiz_knowledge_creation.pdf

Nonaka I. (1991). The knowledge-creating company. Harvard Business Review, 69(6), 96-104. Disponível em:

Nonaka I., \& Takeuchi H. (1995). The knowledge creating company: how Japanese companies create the dynamics of innovation. New York: Oxford University Press.

Rang, H. P., Ritter, J. M., Flower, R. J., \& Henderson, G. (2007). Farmacologia. Rio de Janeiro: Elsevier.

Rasoulinezhad, E. (2011). Measuring the Role of Knowledge Management Processes in the Commercial Banks of Iran. The Electronic Journal of Knowledge Management, 9(4):353-364. Disponível em: http://www.ejkm.com/issue/download.html?idArticle=300.

Santos Júnior, A. B., Bispo, F. C. S., \& Abbad, G. S. (2014). Transferência de treinamento: estudo de um curso de ambientação para novos colaboradores. Resende (RJ): SEGeT.

Santos, L. A., \& Souza, M. A. (2014). O impacto da Anomia Organizacional sobre a motivação para aprender no contexto de uma Instituição Federal do Ensino Superior de um estado do sudeste brasileiro. Boletim Academia Paulista de Psicologia, 34(87), 387-406. Disponível em: http://pepsic.bvsalud.org/scielo.php?script=sci_arttext\&pid=S1415-711X2014000200007.

Scorsolinl-Comin F., Inocente, D. F., \& Miura, I. K. (2011). Aprendizagem organizacional e gestão do conhecimento: Pautas para a gestão de pessoas. Revista Brasileira de Orientação

Rev. Psicol Saúde e Debate. Dez., 2020:6(2): 430-442. 
Profissional, 2 (2), 227-239. Disponível em: http://pepsic.bvsalud.org/scielo.php?script=sci_arttext\&pid=S1679-33902011000200010.

Senge, P. M. (1991). The fifth discipline, the art and practice of the learning organization, Performance \& Instruction, 30 (5), 37.

Silva Junior, A. F. (2007). O setor de serviços e sua importância na economia brasileira. In: Congresso Virtual Brasileiro de Administração. 7 a 15 de dezembro de 2007.

Souza, Y. S. (2004). Organizações de aprendizagem ou aprendizagem organizacional. RAEeletrônica, 3 (1),1-16. Disponível em: http://www.scielo.br/pdf/raeel/v3n1/v3n1a08.

Vaz, C., Inomata, D., Viegas, C., Selig, P., \& Varvakis, G. (2015). Capital intelectual: classificação, formas de mensuração e questionamento sobre usos futuros. Navus - Revista de Gestão e Tecnologia, 5 (2), 73-92. doi:https://doi.org/10.22279/navus.2015.v5n2.p73-92.253 